\title{
Field-induced tunneling rates, polarizabilities, and hyperpolarizabilities for low-lying excited states of $\mathrm{Li}$ and $\mathrm{Na}$
}

\author{
Spyros I. Themelis and Cleanthes A. Nicolaides \\ Theoretical and Physical Chemistry Institute, National Hellenic Research Foundation, \\ 48 Vas. Constantinou Ave., 11635 Athens, Greece \\ (Received 8 March 1994; revised manuscript received 19 December 1994)
}

\begin{abstract}
We have obtained solutions from the state-specific complex eigenvalue Schrödinger equation (CESE) for the dc-field-induced energy shifts and widths of the Li $1 s^{2} 3 s^{2} S, 3 p^{2} P^{o}, 3 d^{2} D$, Na $K L 3 s^{2} S, 3 p^{2} P^{o}$, $4 s^{2} S$, and $3 d^{2} D$ states. These allowed us to compute tunneling rates and scalar and tensor polarizabilities and hyperpolarizabilities. Our values for the polarizabilities are compared with earlier theoretical and experimental ones. We have also computed the tunneling rates using a previously published semiclassical formula and have compared the $a b$ initio results with the semiclassical ones. Due to electronic structure and spectral effects, quantitative as well as qualitative differences occur.
\end{abstract}

PACS number(s): $32.60 .+\mathrm{i}$

\section{INTRODUCTION}

We have recently presented [1-3] a method of $a b$ initio calculation of dc-field-induced tunneling rates (FITR's) and linear and nonlinear polarizabilities, which takes into account the electronic-structure characteristics of each state of interest. The applications involved the ground states of prototype neutral atoms $(\mathrm{He}, \mathrm{Li})$, and negative ions $\left(\mathrm{H}^{-}, \mathrm{Li}^{-}\right)$, as well as the $\mathrm{Li} 1 s^{2} 2 p^{2} \boldsymbol{P}^{o}$ excited state. Furthermore, comparison was made [3] with results obtained from models which, in the absence of results of $a b$ initio quantum-mechanical theory, have been used over the years for the computation of tunneling rates induced by dc or low-frequency fields [4-9].

Motivated by the interest of experimentalists in measuring FITR's [10] and LoSurdo-Stark shifts [11], we have now applied the same theory to the calculation of FITR's and of hyperpolarizabilities for low-lying excited states of $\mathrm{Li}$ and $\mathrm{Na}$, namely, the $\mathrm{Li} 1 s^{2} 3 s{ }^{2} S, 1 s^{2} 3 p^{2} P^{o}$, and $1 s^{2} 3 d^{2} D$, and the $\mathrm{Na} K L 3 s^{2} S, 3 p^{2} P^{o}, 4 s^{2} S$, and $3 d^{2} D$.

Such information was not available on these states until now. As before [3], we have also tested the models in order to provide information about the degree and the range of their validity.

The physics of the application of an external dc field of laboratory strength to states of the alkali-metal atoms is characterized mainly by two types of effects. The first is the field-induced mixing of states of different symmetry. The second is the core polarization induced by the outer electron which affects the outer orbitals and the dipole matrix elements. This effect is now taken into account for the dipole matrix element by an indirect method based on the use of core polarizability $[12,13]$.

The overall calculation involves the solution of the complex eigenvalue Schrödinger equation (CESE) by the state-specific method ([1],[2], and references therein), whereby both the energy shifts and widths, related to the linear and nonlinear polarizabilities and the tunneling rates correspondingly, are obtained.

\section{DESCRIPTION OF THE CALCULATION}

The solution of the complex eigenvalue Schrödinger equation $[1,2]$,

$$
\left(H-z_{0}\right) \psi=0 \text {, }
$$

where $H=H_{0}+\sum_{i} \mathbf{F} \cdot \mathbf{r}_{i}$ is the total Hamiltonian and $z_{0}=E_{0}+\Delta(F)-(i / 2) \Gamma(F)$, was done as follows. The wave function $\psi$ was projected on a function space divided into two nonorthonormal parts: the $Q$ space contains configurations belonging to the bound spectrum of the field-free alkali-metal atoms while the $\boldsymbol{P}$ space contains configurations with complex orbitals representing the high-lying states of the discrete and continuous spectrum. The $\varepsilon l$ continuum orbitals were discretized on a basis of complex STO's as

$$
\phi_{\varepsilon l}=\sum_{n} \alpha_{n} \phi_{n l}(r, \theta),
$$

where

$$
\phi_{n l}(r, \theta)=r^{n+l-1} e^{-\alpha r e^{-i \theta}} .
$$

The complex generalized eigenvalue problem

$$
\left\{\begin{array}{ll}
H_{Q Q} & H_{Q P} \\
H_{P Q} & H_{P P}
\end{array}\right\}\left\{\begin{array}{l}
C_{Q} \\
C_{P}
\end{array}\right]=z\left\{\begin{array}{cc}
I & S_{Q P} \\
S_{P Q} & I
\end{array}\right\}\left\{\begin{array}{l}
C_{Q} \\
C_{P}
\end{array}\right)
$$

was solved repeatedly by varying $\alpha$ and $\theta$ until the state of interest was identified with a stabilized eigenvalue. In order to take into account core polarization effects we have followed the work of $[12,13]$ and have replaced the dipole operator entering in the calculation of matrix elements of $H$ by

$$
D^{(1)}=\left\{1-\frac{\alpha_{d}}{r^{3}} W_{3}\left(r / r_{c}\right)\right\} \mathbf{r},
$$

where $\alpha_{d}$ is the polarizability of the core state while the superscript denotes that the dipole operator is a spherical tensor of rank $1 . W_{n}(x)=1-e^{-x^{n}}$ is a cutoff function 
and $r_{c}$ is an effective core radius.

We also carried out calculations of FITR's using previously published semiclassical formulas. The corrected version of Ilkov et al. [14] gives the tunneling rate as

$$
\begin{aligned}
& \Gamma=C_{n}^{2} l^{*} E_{p} \frac{(2 l+1)(l+|m|) !}{2^{|m|}(|m| !)(l-|m|) !} \\
& \times\left\{\frac{2}{F}\left(2 E_{p}\right)^{3 / 2}\right\}^{2 n^{*}-|m|-1} \exp \left\{-\frac{2}{3} \frac{\left(2 E_{p}\right)^{3 / 2}}{F}\right\} \\
& C_{n^{*} l^{*}}^{2}=\frac{1}{2 \pi n^{*}}\left[\frac{4 e^{2}}{n^{* 2}-l^{* 2}}\right]^{n^{*}}\left[\frac{n^{*}-l^{*}}{n^{*}+l^{*}}\right]^{l^{*}+1 / 2}
\end{aligned}
$$

where $n^{*}=n-\delta_{l}, l^{*}=l-\delta_{1}$ are effective quantum numbers for the state of interest, $\delta_{l}=n-Z^{*} / \sqrt{2 E_{p}}, Z^{*}$ is the effective charge for the escaping electron, and $E_{p}$ is the ionization potential. For the present work $Z^{*}$ was set equal to 1 while the value of $E_{p}$ for each state was computed ab initio.

For $\mathrm{Li}$ the $Q$ space contained the Hartree-Fock (HF) functions $1 s^{2} n l^{2} L, n=2-5, l=0, \ldots, n-1$ while the $P$ space contained configurations of the form $1 s^{2} \varepsilon l$, $l=0,1,2, \ldots$. We used $10 s, 10 p, 10 d, 8 f, 6 g, 4 h$, and $4 i$ complex STO's which were orthgonalized to the core orbitals. Their exponent was optimal at the value $\alpha_{0}=0.25$. For the core polarization correction to dipole operator we used $\alpha_{d}\left(\mathrm{Li}^{+}\right)=0.1884, r_{c}=1.426$.

For $\mathrm{Na}$ the $Q$ space contained the HF configurations $K L n l^{2} L, n=3-6, l=0, \ldots, n-1$, while the same number of complex STO's was used for the $P$ space. The exponent of STO's was optimal at $\alpha_{0}=0.3$ for $K L 3 d^{2} D$ and at $\alpha_{0}=0.4$ for all other states. For the dipole operator we used $\alpha_{d}\left(\mathrm{Na}^{+}\right)=0.946, r_{c}=2.35[13]$.

\section{RESULTS}

\section{A. Lithium}

We have made calculations for the $1 s^{2} 3 s, 1 s^{2} 3 p$, and $1 s^{2} 3 d$ states, including all $m$ components, in a range of field strengths $0 \rightarrow 0.002$ a.u., which corresponds to a maximum value of about $10 \mathrm{MV} / \mathrm{cm}$.

The energy of the $1 s^{2} 3 s$ state decreases as the field strength increases. Figure 1 shows that the energy shift remains almost quadratic for all the values of $F$. Also the energies of the $1 s^{2} 3 p, m=0, \pm 1$ states decrease for small $F$. However, at $F \approx 0.0015$ a.u. the strong coupling of the $m=0$ state with the lower-lying $1 s^{2} 3 s$ state causes an increase of its energy, so that this state comes above the $m= \pm 1$ components, while for larger field strengths it moves to lower values again (Fig. 1). The $1 s^{2} 3 d$ state is split into three components with $m=0, \pm 1, \pm 2$. The $m=0$ and $m= \pm 1$ states are shifted upwards, interacting strongly with the corresponding $1 s^{2} 3 p$ states, while the $m= \pm 2$ state is shifted downwards. The $m=0$ state is the most affected by the field. Its energy shift shows a small "kink" at $F \approx 0.0009$ a.u. coming possibly from a rearrangement of the higher Rydberg states. Also, there is an avoided crossing at $F \approx 0.0016$ a.u., which pushes it

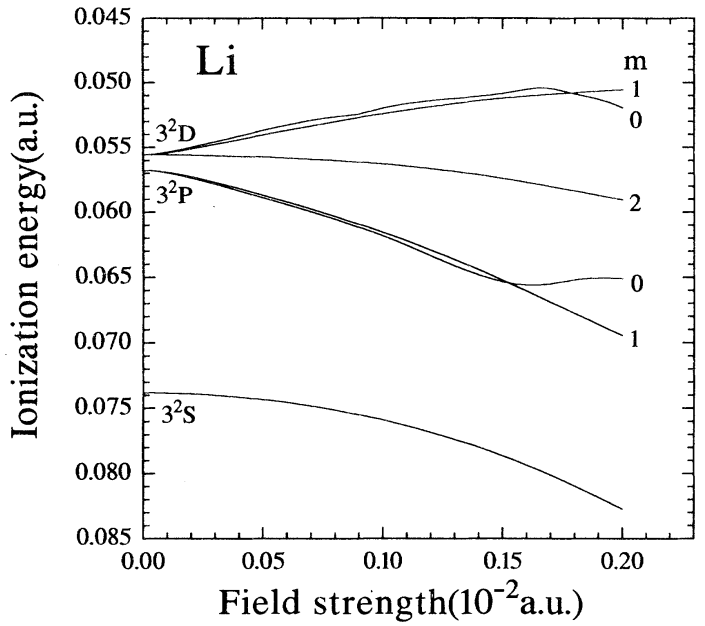

FIG. 1. Lithium Stark structure for $|m|=0,1,2$. There is strong interaction for states having $m=0$ whose position with respect to that of the $m= \pm 1$ levels changes after field strength of about $0.15 \times 10^{-2}$ a.u. The polarizability of the $1 s^{2} 3 d$ $m=0, \pm 1$ states is negative.

below the $m= \pm 1$ component (Fig. 1). As regards the tunneling rates for the states of $\mathrm{Li}$, they are presented in Tables I-III together with the results of Eq. (5). The semiclassical (SC) results for $1 s^{2} 3 s$ and $1 s^{2} 3 p$ are larger than the quantum mechanical (QM) and particularly for strong fields there is a considerable difference of orders of magnitude. For $1 s^{2} 3 d^{2} D, m=0$, there is a good agreement with semiclassical results for small field strengths, but for $F>0.0009$ a.u. the difference becomes large [see Fig. 2(a)]. Almost the same picture is seen for $1 s^{2} 3 d^{2} D$, $m= \pm 1$ [Fig. 2(b)], while the results for $m= \pm 2$ differ by an order of magnitude for all values of $F$. In summary, we can say that the difference between the $a b$ initio quantum-mechanical results and those from Eq. (5) becomes large for high $F$ and for high $m$.

TABLE I. Field-induced tunneling widths for $1 s^{2} 3 s^{2} S$ calculated from Eq. (5) and from the present ab initio polyelectronic theory. The difference increases with increasing field strength. Numbers in square brackets denote powers of 10 .

\begin{tabular}{ccc}
\hline \hline & \multicolumn{2}{c}{$\Gamma / 2$ (a.u.) } \\
\cline { 2 - 3 }$F\left(10^{-2}\right.$ a.u.) & Ab initio & Semiclassical \\
\hline 0.10 & $5.0[-10]$ & $1.92[-9]$ \\
0.11 & $8.70[-9]$ & $3.99[-8]$ \\
0.12 & $9.03[-8]$ & $4.86[-7]$ \\
0.13 & $6.08[-7]$ & $3.91[-6]$ \\
0.14 & $2.90[-6]$ & $2.29[-5]$ \\
0.15 & $1.05[-5]$ & $1.04[-4]$ \\
0.16 & $3.00[-5]$ & $3.81[-4]$ \\
0.17 & $7.12[-5]$ & $1.19[-3]$ \\
0.18 & $1.44[-4]$ & $3.21[-3]$ \\
0.19 & $2.56[-4]$ & $7.71[-3]$ \\
0.20 & $4.11[-4]$ & $1.68[-2]$ \\
\hline \hline
\end{tabular}


TABLE II. As in Table I for $\mathrm{Li} 1 s^{2} 3 p^{2} P^{o}, m=0, m= \pm 1$. The difference between the two calculations is large for all values of $F$. Numbers in square brackets denote powers of 10 .

\begin{tabular}{ccccc}
\hline \hline & \multicolumn{3}{c}{$\Gamma / 2$ (a.u.) } \\
\cline { 2 - 3 }$F\left(10^{-2}\right.$ a.u. $)$ & Ab initio & Semiclassical & & \multicolumn{2}{c}{ Ab initio } \\
\cline { 2 - 5 } \cline { 4 - 5 } 0.060 & $2.0[-10]$ & $7.29[-10]$ & & \\
0.065 & $9.0[-10]$ & $1.29[-8]$ & & \\
0.070 & $5.90[-9]$ & $1.48[-7]$ & & \\
0.075 & $3.92[-8]$ & $1.19[-6]$ & & \\
0.080 & $2.19[-7]$ & $7.27[-6]$ & $2.20[-9]$ & \\
0.090 & $3.39[-6]$ & $1.41[-4]$ & $5.32[-8]$ & $7.60[-8]$ \\
0.100 & $2.59[-5]$ & $1.42[-3]$ & $5.61[-7]$ & $1.65[-6]$ \\
0.110 & $1.13[-4]$ & $9.03[-3]$ & $3.38[-6]$ & $1.86[-5]$ \\
0.120 & $3.25[-4]$ & $4.06[-2]$ & $1.37[-5]$ & $1.30[-4]$ \\
0.130 & $6.98[-4]$ & $1.40[-1]$ & $4.08[-5]$ & $6.37[-4]$ \\
0.140 & $1.24[-3]$ & $3.95[-1]$ & $9.57[-5]$ & $2.38[-3]$ \\
0.150 & $1.93[-3]$ & $9.47[-1]$ & $1.88[-4]$ & $7.23[-3]$ \\
0.160 & $2.71[-3]$ & 1.99 & $3.21[-4]$ & $1.86[-2]$ \\
0.170 & $3.40[-3]$ & 3.78 & $4.97[-4]$ & $4.17[-2]$ \\
0.180 & $3.69[-3]$ & 6.55 & $7.13[-4]$ & $8.39[-2]$ \\
0.190 & $3.69[-3]$ & $1.06[1]$ & $9.66[-4]$ & $1.54[-1]$ \\
0.200 & $3.61[-3]$ & $1.61[1]$ & $1.25[-3]$ & $2.63[-1]$ \\
\hline \hline
\end{tabular}

\section{B. Sodium}

We have made calculations for all the $m$ components of the $(K L) 3 s,(K L) 3 p,(K L) 4 s$, and $(K L) 3 d$ states. The situation here is somewhat different than for $\mathrm{Li}$, since the $4 s$ state is lying lower than the $3 d$ one. Aspects of the field-perturbed low-lying spectra of $\mathrm{Li}$ and $\mathrm{Na}$ are dis- cussed in Ref. [11].

Due to their large ionization potential, the response of the $3^{2} S$ and $3{ }^{2} P^{o}$ states to an experimentally attainable field strength is very small. Therefore we extended our calculation for $3^{2} S$ to $F=1.2 \times 10^{-2}$ a.u. and for $3{ }^{2} P^{o}$ to $F=6.0 \times 10^{-3}$ a.u. in order to check the accuracy of the results of Eq. (5). The LoSurdo-Stark shift of these states

TABLE III. As in Table I for Li $1 s^{2} 3 d^{2} D, m=0, \pm 1, \pm 2$. The difference for $m=0$ becomes large after $F>0.09 \times 10^{-2}$ a.u. Numbers in square brackets denote powers of 10 .

\begin{tabular}{|c|c|c|c|c|c|c|}
\hline \multirow[b]{3}{*}{$F\left(10^{-2}\right.$ a.u. $)$} & \multicolumn{6}{|c|}{$\Gamma / 2$ (a.u.) } \\
\hline & \multicolumn{2}{|c|}{$m=0$} & \multicolumn{2}{|c|}{$m= \pm 1$} & \multicolumn{2}{|c|}{$m= \pm 2$} \\
\hline & $A b$ initio & Semiclassical & $A b$ initio & Semiclassical & $A b$ initio & Semiclassical \\
\hline 0.055 & $5.00[-10]$ & $3.81[-11]$ & & & & \\
\hline 0.060 & $2.70[-9]$ & $1.04[-9]$ & & & & \\
\hline 0.065 & $4.24[-8]$ & $1.65[-8]$ & $1.0[-10]$ & $4.4[-10]$ & & \\
\hline 0.070 & $5.14[-7]$ & $1.72[-7]$ & $6.0[-10]$ & $4.9[-9]$ & & \\
\hline 0.075 & $4.33[-6]$ & $1.28[-6]$ & $3.9[-9]$ & $3.89[-8]$ & & \\
\hline 0.080 & $2.57[-5]$ & $7.27[-6]$ & $2.35[-8]$ & $2.35[-7]$ & & \\
\hline 0.085 & $1.05[-4]$ & $3.30[-5]$ & $1.10[-7]$ & $1.14[-6]$ & & \\
\hline 0.090 & $2.71[-4]$ & $1.25[-4]$ & $3.94[-7]$ & $4.54[-6]$ & & \\
\hline 0.095 & $4.53[-4]$ & $4.11[-4]$ & $1.18[-6]$ & $1.55[-5]$ & $3.17[-8]$ & $1.99[-7]$ \\
\hline 0.100 & $5.60[-4]$ & $1.14[-3]$ & $2.94[-6]$ & $4.63[-5]$ & $1.09[-7]$ & $6.25[-7]$ \\
\hline 0.110 & $7.21[-4]$ & $6.70[-3]$ & $1.02[-5]$ & $2.99[-4]$ & $8.07[-7]$ & $4.43[-6]$ \\
\hline 0.120 & $9.68[-4]$ & $2.82[-2]$ & $1.86[-5]$ & $1.37[-3]$ & $3.95[-6]$ & $2.22[-5]$ \\
\hline 0.130 & $1.38[-3]$ & $9.19[-2]$ & $2.49[-5]$ & $4.84[-3]$ & $1.39[-5]$ & $8.49[-5]$ \\
\hline 0.140 & $2.02[-3]$ & $2.47[-1]$ & $3.28[-5]$ & $1.40[-2]$ & $3.72[-5]$ & $2.64[-4]$ \\
\hline 0.150 & $2.97[-3]$ & $5.66[-1]$ & $4.93[-5]$ & $3.44[-2]$ & $8.16[-5]$ & $6.96[-4]$ \\
\hline 0.160 & $4.39[-3]$ & 1.15 & $8.22[-5]$ & $7.43[-2]$ & $1.53[-4]$ & $1.61[-3]$ \\
\hline 0.170 & $6.50[-3]$ & 2.10 & $1.37[-4]$ & $1.45[-1]$ & $2.54[-4]$ & $3.32[-3]$ \\
\hline 0.180 & $8.64[-3]$ & 3.54 & $2.15[-4]$ & $2.58[-1]$ & $3.85[-4]$ & $6.26[-3]$ \\
\hline 0.190 & $1.08[-2]$ & 5.56 & $3.15[-4]$ & $4.28[-1]$ & $5.46[-4]$ & $1.10[-2]$ \\
\hline 0.200 & $1.30[-2]$ & 8.23 & $4.36[-4]$ & $6.67[-1]$ & $7.33[-4]$ & $1.80[-2]$ \\
\hline
\end{tabular}



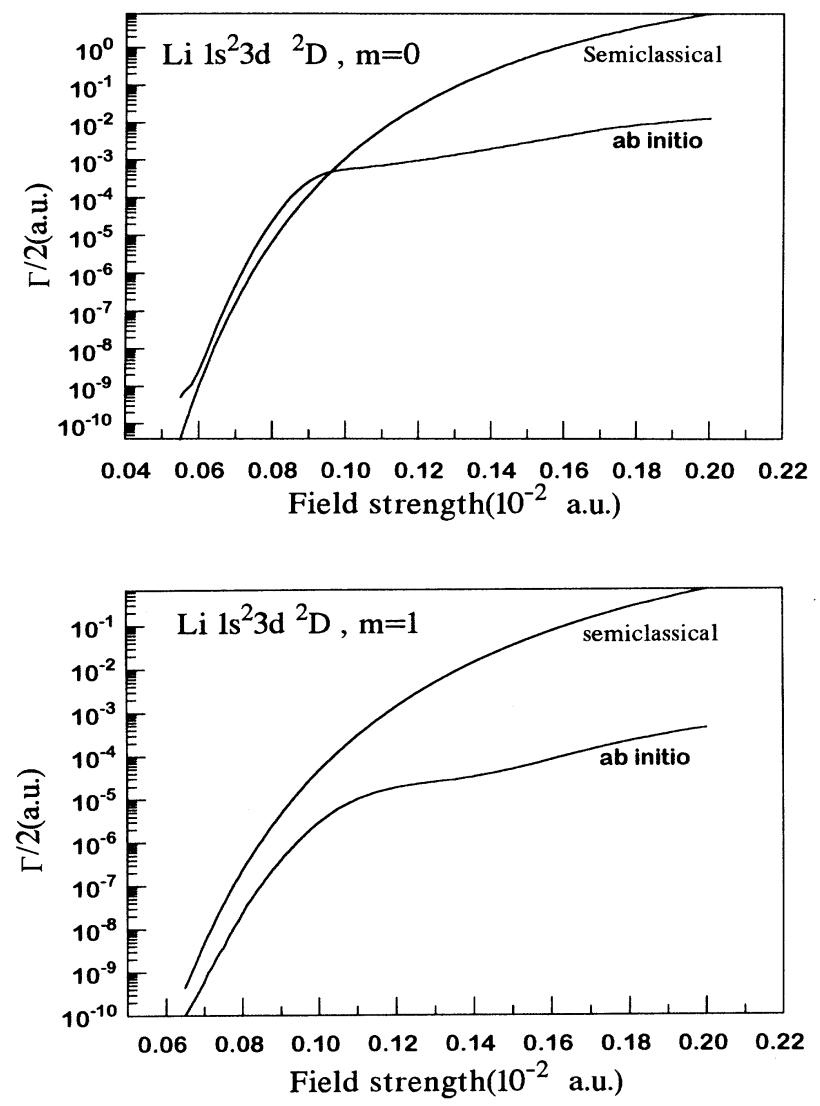

FIG. 2. (a) Field-induced tunneling width for $\mathrm{Li} 1 s^{2} 3 d^{2} D$, $m=0$ calculated from Eq. (5) and from the present polyelectronic theory. The difference between the two curves becomes large after $F>0.09 \times 10^{-2}$ a.u. (b) As in (a) for $\mathrm{Li} 1 s^{2} 3 d^{2} D$, $m= \pm 1$.

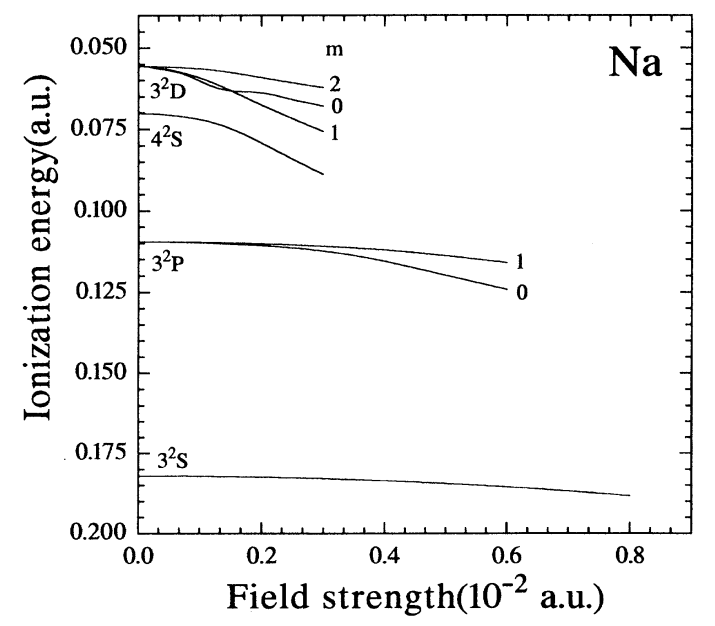

FIG. 3. Sodium Stark structure for $|m|=0,1,2$. The $3^{2} P^{o}$ $m=0, \pm 1$ states have different polarizabilities. Also, the ordering of $3^{2} D, m=0, m= \pm 1$ states changes for $F>0.0015$ a.u., a result of strong interaction of the $m=0$ level with the lowerlying level corresponding to the $4{ }^{2} S$ field-free level.

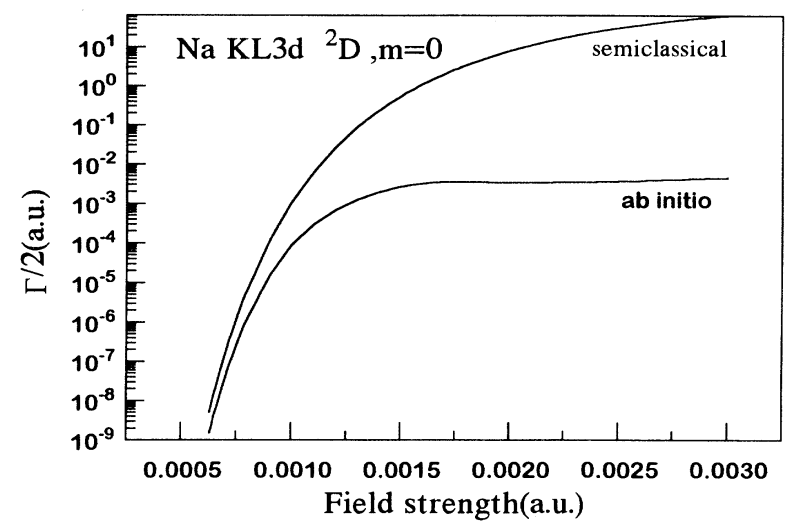

FIG. 4. As in Fig. 2(a) for $\mathrm{Na} K L 3 d^{2} D, m=0$. The quantum-mechanical tunneling rate stabilizes for $F>0.0016$ a.u.

is shown in Fig. 3. For $K L 3 s$ the energy shift is almost quadratic in $F$ and quite small $\left(\Delta=-1.5 \times 10^{-2}\right.$ a.u. at $F=1.2 \times 10^{-2}$ a.u.). The $K L 3 p$ is split into two components. The energy shift of the $m=0$ state is twice as large as that of the $m= \pm 1$ state (see Fig. 3) and for large values of $F$ this state has an energy shift almost linear in $F$. Note that the same was found in the $\mathrm{Li} 1 s^{2} 2 p^{2} P^{o}$ state [2]. Also the same is met for $K L 4 s$, which, having a smaller ionization potential than the $3 \mathrm{~s}$ state, has significant energy shift $\left(\Delta=-1.863 \times 10^{-2}\right.$ a.u. at $F=3.0 \times 10^{-3}$ a.u.). The $K L 3 d$ state is split into three components, all shifted downwards for small $F$. At about $F \sim 0.0015$ a.u., the field-dressed $m=0$ level is pushed up by the strong interaction with the field-dressed, lowerlying $K L 4 s$ state, and its position comes above the $m= \pm 1$ level. However, its energy decreases again. The tunneling rates for the states of $\mathrm{Na}$ are presented on Tables IV-VII. For $3{ }^{2} S$ there is relatively good agree-

TABLE IV. As in Table I for $\mathrm{Na} K L 3 s^{2} S$. Here, the difference even for large field strength does not exceed a factor of 5 . Numbers in square brackets denote powers of 10 .

\begin{tabular}{ccc}
\hline \hline & \multicolumn{2}{c}{$\Gamma / 2$ (a.u.) } \\
\cline { 2 - 3 }$F\left(10^{-2}\right.$ a.u.) & Ab initio & Semiclassical \\
\hline 0.50 & $1.40[-9]$ & $2.33[-9]$ \\
0.55 & $1.58[-8]$ & $2.68[-8]$ \\
0.60 & $1.12[-7]$ & $2.02[-7]$ \\
0.65 & $5.64[-7]$ & $1.10[-6]$ \\
0.70 & $2.19[-6]$ & $4.62[-6]$ \\
0.75 & $6.91[-6]$ & $1.59[-5]$ \\
0.80 & $1.83[-5]$ & $4.65[-5]$ \\
0.85 & $4.22[-5]$ & $1.19[-4]$ \\
0.90 & $8.64[-5]$ & $2.71[-4]$ \\
0.95 & $1.60[-4]$ & $5.63[-4]$ \\
1.00 & $2.72[-4]$ & $1.08[-3]$ \\
1.05 & $4.31[-4]$ & $1.94[-3]$ \\
1.10 & $6.42[-4]$ & $3.28[-3]$ \\
1.15 & $9.10[-4]$ & $5.29[-3]$ \\
1.20 & $1.24[-3]$ & $8.15[-3]$ \\
\hline \hline
\end{tabular}


TABLE V. As in Table IV for Na $K L 3 p^{2} P^{o}, m=0, m= \pm 1$. Numbers in square brackets denote powers of 10 .

\begin{tabular}{|c|c|c|c|c|}
\hline \multirow[b]{3}{*}{$F\left(10^{-2}\right.$ a.u. $)$} & \multicolumn{4}{|c|}{$\Gamma / 2$ (a.u.) } \\
\hline & \multicolumn{2}{|c|}{$m=0$} & \multicolumn{2}{|c|}{$m= \pm 1$} \\
\hline & $A b$ initio & Semiclassical & $A b$ initio & Semiclassical \\
\hline 0.210 & $3.10[-9]$ & $1.48[-8]$ & & \\
\hline 0.230 & $3.64[-8]$ & $1.86[-7]$ & & \\
\hline 0.250 & $2.76[-7]$ & $1.53[-6]$ & $2.90[-9]$ & $1.86[-8]$ \\
\hline 0.275 & $2.15[-6]$ & $1.34[-5]$ & $2.91[-8]$ & $1.80[-7]$ \\
\hline 0.300 & $1.11[-5]$ & $7.99[-5]$ & $1.84[-7]$ & $1.17[-6]$ \\
\hline 0.325 & $4.11[-5]$ & $3.55[-4]$ & $8.44[-7]$ & $5.62[-6]$ \\
\hline 0.350 & $1.17[-4]$ & $1.25[-3]$ & $2.98[-6]$ & $2.13[-5]$ \\
\hline 0.375 & $2.70[-4]$ & $3.66[-3]$ & $8.57[-6]$ & $6.70[-5]$ \\
\hline 0.400 & $5.29[-4]$ & $9.26[-3]$ & $2.09[-5]$ & $1.81[-4]$ \\
\hline 0.425 & $9.05[-4]$ & $2.08[-2]$ & $4.45[-5]$ & $4.30[-4]$ \\
\hline 0.450 & $1.41[-3]$ & $4.20[-2]$ & $8.46[-5]$ & $9.23[-4]$ \\
\hline 0.475 & $2.04[-3]$ & $7.83[-2]$ & $1.47[-4]$ & $1.82[-3]$ \\
\hline 0.500 & $2.80[-3]$ & $1.36[-1]$ & $2.36[-4]$ & $3.32[-3]$ \\
\hline 0.525 & $3.67[-3]$ & $2.22[-1]$ & $3.57[-4]$ & $5.69[-3]$ \\
\hline 0.550 & $4.65[-3]$ & $3.45[-1]$ & $5.10[-4]$ & $9.25[-3]$ \\
\hline 0.575 & $5.95[-3]$ & $5.11[-1]$ & $6.97[-4]$ & $1.44[-2]$ \\
\hline 0.600 & $6.96[-3]$ & $7.30[-1]$ & $9.19[-4]$ & $2.14[-2]$ \\
\hline
\end{tabular}

ment between the QM and SC results, while a significant difference is observed for $4^{2} S$ and $3^{2} P^{o}$, especially for the $m= \pm 1$ levels. For the $3^{2} D, m=0$ state, the FITR remains almost constant at a value of about $\Gamma \sim 7 \times 10^{-3}$ a.u. (see Fig. 4), after the value of $F$ where this state is pushed up. The agreement of the QM tunneling rates with the SC ones is good for small values of the field strength but not for large values. The same is true for the $m= \pm 1$ and $m= \pm 2$ states [see Table VII]. These levels have considerable FITR's for values of the field strength of the order 3-10 MV/cm, which can be attained in the laboratory.

The poor agreement between our ab initio calculation and the semiclassical formula (5) for $\mathrm{Li}$ and for $\mathrm{Na}$ cannot be eliminated by changing the computed values of the

TABLE VI. As in Table IV for Na $K L 4 s^{2} S$. Numbers in square brackets denote powers of 10 .

\begin{tabular}{ccc}
\hline & \multicolumn{2}{c}{$\Gamma / 2$ (a.u.) } \\
\cline { 2 - 3 }$F\left(10^{-2}\right.$ a.u. $)$ & Ab initio & Semiclassical \\
\hline 0.096 & $3.60[-9]$ & $7.93[-9]$ \\
0.100 & $1.33[-8]$ & $2.96[-8]$ \\
0.104 & $3.98[-8]$ & $9.30[-8]$ \\
0.108 & $1.00[-7]$ & $2.75[-7]$ \\
0.112 & $2.49[-7]$ & $7.48[-7]$ \\
0.116 & $5.81[-7]$ & $1.89[-6]$ \\
0.120 & $1.27[-6]$ & $4.47[-6]$ \\
0.125 & $3.09[-6]$ & $1.20[-5]$ \\
0.150 & $7.54[-5]$ & $5.84[-4]$ \\
0.175 & $4.48[-4]$ & $8.43[-3]$ \\
0.200 & $1.26[-3]$ & $5.77[-2]$ \\
0.225 & $2.47[-3]$ & $2.43[-1]$ \\
0.250 & $3.98[-3]$ & $7.30[-1]$ \\
0.275 & $5.75[-3]$ & 1.73 \\
0.300 & $7.73[-3]$ & 3.42 \\
\hline \hline
\end{tabular}

ionization potential by the experimentally known ones or by parameters "appropriately" chosen in order to give "correct" results. The large difference occurring for values of the field strength that give crossings and interaction with nearby lying states is not removed in such a way. This result is relevant to research where the values of $\Gamma(F)$ given by Eq. (5) are used for the calculation of tunneling ionization in the quasistatic approximation to laser-atom interactions [7,8].

\section{POLARIZABILITIES AND HYPERPOLARIZABILITIES}

For small field strengths the energy shift is expanded as

$$
\Delta_{L M}(F)=-\frac{1}{2 !} \alpha_{L M} F^{2}-\frac{1}{4 !} \gamma_{L M} F^{4} \cdots,
$$

where $\alpha_{L M}$ is the polarizability and $\gamma_{L M}$ is the hyperpolarizability of the state $(L, M)$. These quantities are obtained by fitting 40 calculated values of $\Delta_{L M}(F)$ to a tenth degree polynomial. The $2 \times L+1$ polarizabilities and hyperpolarizabilities of an atomic state with angular momentum $L$ are not all independent since they can be expressed as

TABLE VIII. Calculated scalar and tensor parts of the polarizabilities and hyperpolarizabilities for $\mathrm{Li}$ states. All results are in a.u. Numbers in square brackets denote powers of 10.

\begin{tabular}{lccc}
\hline & $1 s^{2} 3 s$ & $1 s^{2} 3 p$ & $1 s^{2} 3 d$ \\
\hline$\alpha_{0}$ & 4136 & 37557 & -20468 \\
$\alpha_{2}$ & & -3071 & 21944 \\
$\gamma_{0}$ & $8.69[8]$ & $-6.02[12]$ & $4.56[12]$ \\
$\gamma_{2}$ & & $1.04[12]$ & $-3.97[12]$ \\
$\gamma_{4}$ & & & $0.195[12]$ \\
\hline
\end{tabular}


TABLE VII. As in Table IV for Na $K L 3 d^{2} D, m=0, \pm 1, \pm 2$. For $m=0$ the quantum-mechanical tunneling rate stabilizes for $F>0.0016$ a.u. Numbers in square brackets denote powers of 10 .

\begin{tabular}{|c|c|c|c|c|c|c|}
\hline \multirow[b]{3}{*}{$F\left(10^{-2}\right.$ a.u. $)$} & \multicolumn{6}{|c|}{$\Gamma / 2$ (a.u.) } \\
\hline & \multicolumn{2}{|c|}{$m=0$} & \multicolumn{2}{|c|}{$m= \pm 1$} & \multicolumn{2}{|c|}{$m= \pm 2$} \\
\hline & $A b$ initio & Semiclassical & $A b$ initio & Semiclassical & $A b$ initio & Semiclassical \\
\hline 0.065 & $4.20[-9]$ & $1.47[-8]$ & & & & \\
\hline 0.070 & $3.79[-8]$ & $1.55[-7]$ & & & & \\
\hline 0.075 & $2.53[-7]$ & $1.16[-6]$ & $4.20[-9]$ & $3.51[-8]$ & & \\
\hline 0.080 & $1.25[-6]$ & $6.62[-6]$ & $2.71[-8]$ & $2.14[-7]$ & & \\
\hline 0.090 & $1.52[-5]$ & $1.15[-4]$ & $4.16[-7]$ & $4.16[-6]$ & $7.20[-9]$ & $5.04[-8]$ \\
\hline 0.100 & $8.81[-5]$ & $1.06[-3]$ & $3.47[-6]$ & $4.28[-5]$ & $1.00[-7]$ & $5.77[-7]$ \\
\hline 0.110 & $2.94[-4]$ & $6.27[-3]$ & $1.70[-5]$ & $2.78[-4]$ & $7.50[-7]$ & $4.12[-6]$ \\
\hline 0.120 & $6.78[-4]$ & $2.65[-2]$ & $5.65[-5]$ & $1.28[-3]$ & $3.69[-6]$ & $2.07[-5]$ \\
\hline 0.130 & $1.24[-3]$ & $8.69[-2]$ & $1.39[-4]$ & $4.56[-3]$ & $1.30[-5]$ & $7.98[-5]$ \\
\hline 0.140 & $1.93[-3]$ & $2.34[-1]$ & $2.78[-4]$ & $1.32[-2]$ & $3.52[-5]$ & $2.49[-4]$ \\
\hline 0.150 & $2.67[-3]$ & $5.40[-1]$ & $4.75[-4]$ & $3.27[-2]$ & $7.78[-5]$ & $6.60[-4]$ \\
\hline 0.160 & $3.27[-3]$ & 1.10 & $7.29[-4]$ & $7.09[-2]$ & $1.47[-4]$ & $1.53[-3]$ \\
\hline 0.170 & $3.55[-3]$ & 2.02 & $1.03[-3]$ & $1.38[-1]$ & $2.45[-4]$ & $3.16[-3]$ \\
\hline 0.180 & $3.56[-3]$ & 3.40 & $1.39[-3]$ & $2.47[-1]$ & $3.73[-4]$ & $5.99[-3]$ \\
\hline 0.190 & $3.50[-3]$ & 5.36 & $1.78[-3]$ & $4.11[-1]$ & $5.30[-4]$ & $1.05[-2]$ \\
\hline 0.200 & $3.46[-3]$ & 7.96 & $2.20[-3]$ & $6.43[-1]$ & $7.15[-4]$ & $1.73[-2]$ \\
\hline 0.250 & $3.75[-3]$ & $3.11[1]$ & $4.75[-3]$ & 3.14 & $1.96[-3]$ & $1.07[-1]$ \\
\hline 0.300 & $4.63[-3]$ & $6.52[1]$ & $7.78[-3]$ & 7.90 & $3.56[-3]$ & $3.19[-1]$ \\
\hline
\end{tabular}

$$
\begin{aligned}
& \alpha_{L M}=\alpha_{0}+\alpha_{2} \frac{3 M^{2}-L(L+1)}{L(2 L-1)}, \\
& \gamma_{L M}=\gamma_{0}+\gamma_{2} \frac{3 M^{2}-L(L+1)}{L(2 L-1)}+\gamma_{4} \frac{35 M^{4}+\{25-30 L(L+1)\} M^{2}+(L-1) L(L+1)(L+2)}{L(2 L-1)(2 L-2)(2 L-3)},
\end{aligned}
$$

where $\alpha_{0}$ and $\alpha_{2}$ are the scalar and tensor parts of the dipole polarizability and $\gamma_{0}$ and $\gamma_{2}$ and $\gamma_{4}$ are the scalar and tensor parts of the dipole hyperpolarizability corresponding to tensors of rank 2 and 4, respectively [15]. The calculation of $\alpha_{k}$ and $\gamma_{k}$ can be done by inverting the expressions (7a) and (7b).

Our results for $\mathrm{Li}$ are presented in Table VIII. There is an obvious $m$ dependence of $\alpha_{L M}$ and $\gamma_{L M}$ in magnitude and in sign which gives large anisotropy particularly to $3^{2} D$. Note that for this state the tensor part of $\alpha_{L M}$ is larger than its scalar part. Table IX gives an indication of the accuracy of our result for the $\mathrm{Li} 1 s^{2} 3 s^{2} S$ state by comparing it with other theoretical values [16-18].

The breakdown of $\alpha_{L M}, \gamma_{L M}$ in terms of their scalar and tensor parts for $\mathrm{Na}$ states is given in Table $\mathrm{X}$. The magnitude of the various quantities increases with in-

TABLE IX. Comparison of $\alpha\left(3^{2} S\right)$ of $\mathrm{Li}$ obtained from this work with other available results. All values are in a.u.

\begin{tabular}{ll} 
Ref. [16], lower bound & 3770 \\
Ref. [16] & 4088 \\
Ref. [17], lower bound & 4121 \\
Ref. [18] & 4135 \\
This work & 4136 \\
\hline
\end{tabular}

creasing excitation energy. Table XI compares the calculated values of the polarizabilities of the $\mathrm{Na} K L 3 \mathrm{~s}^{2} S$ and $3 p^{2} P^{o}$ states with other available data [17,19-24]. Finally, Table XII collects the available values for the hyperpolarizability of $3 s$.

\section{CONCLUSION}

We have predicted from $a b$ initio quantum-mechanical calculations the FITR's of a number of low-lying levels of $\mathrm{Li}$ and $\mathrm{Na}$ for experimentally attainable field strengths. These results were used to test the semiclassical formula

TABLE X. Calculated scalar and tensor parts of the polarizabilities and hyperpolarizabilities for $\mathrm{Na}$ states. All results are in a.u. Numbers in square brackets denote powers of 10.

\begin{tabular}{lcccc}
\hline \hline & $3 s$ & $3 p$ & $4 s$ & \multicolumn{1}{c}{$3 d$} \\
\hline$\alpha_{0}$ & 184.5 & 379.3 & 3389.0 & 6155.0 \\
$\alpha_{2}$ & & -86.2 & & -4794.0 \\
& & & & $-4.00[10]$ \\
$\gamma_{0}$ & $1.14[6]$ & $4.09[7]$ & $2.78[9]$ & $3.54[10]$ \\
$\gamma_{2}$ & & $-2.56[7]$ & & $-0.18[10]$ \\
$\gamma_{4}$ & & & & \\
\hline
\end{tabular}


TABLE XI. Comparison of $\alpha\left(3^{2} S\right)$ and $\alpha\left(3^{2} P^{o}\right)$ of $\mathrm{Na}$ obtained from this work with other available results. All values are in a.u.

\begin{tabular}{lccc}
\hline \hline & $\alpha_{0}\left(3^{2} S\right)$ & $\alpha_{0}\left(3^{2} P^{o}\right)$ & $\alpha_{2}\left(3^{2} P^{o}\right)$ \\
\hline $\begin{array}{l}\text { Ref. [17], lower bound } \\
\text { Ref. [19], experiment }\end{array}$ & 162 & & \\
Ref. [20] & 184 & $349 \pm 13$ & $-113 \pm 16$ \\
Ref. [21] & 161 & 383 & \\
Ref. [22] & & 346 & -84 \\
Ref. [23], experiment & $159 \pm 3$ & & \\
Ref. [22], experiment & & 358 & \\
Ref. [24], experiment & & & $-87 \pm 10$ \\
This work & 184 & 379 & -86 \\
\hline
\end{tabular}

(5), which, of course, does not account for electronic structure and for field-induced level interactions. Depending on the case, there are similarities as well as differences, the latter becoming larger with increasing
TABLE XII. Comparison of $\gamma\left(3^{2} S\right)$ of $\mathrm{Na}$ with other theoretical results. All values are in $10^{5}$ a.u.

\begin{tabular}{ll}
\hline \hline Ref. [20] & 10.09 \\
Ref. [25], without core polarization & 14 \\
Ref. [25], inclusion of core polarization & 10.3 \\
This work & 11.4 \\
\hline
\end{tabular}

field strength and for levels with the largest $m$ (see the figures).

The theory also allows the determination of polarizabilities and hyperpolarizabilities (see the tables). The accuracy of these numbers should be slightly better in $\mathbf{L i}$ than in $\mathrm{Na}$, where core single excitations and intershell pair correlations (neglected here) should play a larger role. On the other hand, the effect of the core on the transition matrix elements was taken into account approximately, through Eq. (4).
[1] C. A. Nicolaides and S. I. Themelis, Phys. Rev. A 47, 3122 (1993).

[2] S. I. Themelis and C. A. Nicolaides, Phys. Rev. A 46, R21 (1992).

[3] S. I. Themelis and C. A. Nicolaides, Phys. Rev. A 49, 596 (1994).

[4] Yu. N. Demkov and G. F. Drukarev, Zh. Eksp. Teor. Fiz. 47, 518 (1964) [Sov. Phys. JETP 20, 614 (1965)].

[5] A. M. Perelomov and V. S. Popov, Zh. Eksp. Teor. Fiz. 52, 514 (1967) [Sov. Phys. JETP 25, 336 (1967)].

[6] U. V. Ammosov, N. B. Delone, and V. P. Krainov, Zh. Eksp. Teor. Fiz. 91, 2008 (1986) [Sov. Phys. JETP 64, 1191 (1986)].

[7] P. B. Corkum, N. H. Burnett, and F. Brunel, Phys. Rev. Lett. 62, 1259 (1989).

[8] K. Kondo, T. Tamida, Y. Nabekawa, and S. Watanabe, Phys. Rev. A 49, 3881 (1994).

[9] B. E. Sauer, S. Yoakum, L. Moorman, P. M. Koch, D. Richards, and P. A. Dando, Phys. Rev. Lett. 68, 468 (1992).

[10] A. B. Filuk (private communication).

[11] L. Windholz, M. Musso, G. Zerza, and H. Jäger, Phys. Rev. A 46, 5812 (1992).

[12] I. B. Bersuker, Opt. Spectrosk. 3, 97 (1957).
[13] S. Hameed, A. Herzenberg, and M. G. James, J. Phys. B 1, 822 (1968); J. C. Weisheit, Phys. Rev. A 5, 1621 (1972).

[14] F. A. Ilkov, J. E. Decker, and S. L. Chin, J. Phys. B 25, 4005 (1992).

[15] V. A. Davydkin and V. D. Ovsiannikov, J. Phys. B 19, 2071 (1986).

[16] J. S. Sims, S. A. Hagstrom, and J. R. Rumble, Jr., Phys. Rev. A 14, 576 (1976).

[17] D. R. Beck and C. A. Nicolaides, Chem. Phys. Lett. 49, 357 (1977).

[18] D. V. Ponomarenko and A. F. Shestakov, Chem. Phys. Lett. 210, 269 (1993).

[19] P. Hannaford, W. R. MacGillivary, and M. C. Standage, J. Phys. B 12, 4033 (1979).

[20] P. W. Langhoff, J. D. Lyons, and R. P. Hurst, Phys Rev. 148, 18 (1966).

[21] S. A. Adelman and A. Szabo, J. Chem. Phys. 58, 687 (1973).

[22] R. W. Schmieder, A. Lurio, and W. Happer, Phys. Rev. A 3, 1209 (1971).

[23] R. W. Molof, H. L. Schwartz, T. M. Miller, and B. Bederson, Phys. Rev. A 10, 1131 (1974).

[24] D. Zimmermann, Z. Naturforsch. 252, 196 (1970).

[25] P. Fuentealba and O. Reyes, J. Phys. B 26, 2245 (1993). 